\title{
ArcheoSciences
}

Revue d'archéométrie

\section{La paléogénétique en tant qu'approche archéométrique au cours des 30 dernières années}

Paleogenetics as archeometrical approach over the last 30 years

\section{Eva-Maria Geigl}

\section{(2) OpenEdition}

1 Journals

\section{Édition électronique}

URL : http://journals.openedition.org/archeosciences/5575

DOI : 10.4000/archeosciences. 5575

ISSN : 2104-3728

\section{Éditeur}

Presses universitaires de Rennes

\section{Édition imprimée}

Date de publication : 27 juin 2018

Pagination : 135-144

ISBN : 978-2-7535-7587-5

ISSN : 1960-1360

\section{Référence électronique}

Eva-Maria Geigl, «La paléogénétique en tant qu'approche archéométrique au cours des 30 dernières années », ArcheoSciences [En ligne], 42-1 | 2018, mis en ligne le 27 juin 2020, consulté le 02 janvier 2020. URL : http://journals.openedition.org/archeosciences/5575; DOI : 10.4000/archeosciences. 5575 


\title{
La paléogénétique en tant qu'approche archéométrique au cours des 30 dernières années
}

\author{
Paleogenetics as Archeometrical Approach Over the Last 30 Years
}

\author{
Eva-Maria GeIGL ${ }^{a}$
}

Résumé : La paléogénétique, l'étude génétique des organismes et populations du passé, est devenue possible quand les méthodes de la biologie moléculaire ont permis l'analyse de l'ADN préservé en état très dégradé et en très faible quantité dans certains restes biologiques anciens comme des ossements, des dents, des poils, ou encore des tissus mous de momies. Ces études ont contribué de manière très significative à l'archéologie, la paléontologie, la paléopathologie ou encore la paléoécologie. Plus récemment, le progrès des méthodes de séquençage de l'ADN a conduit à un nouveau saut quantique de ce domaine puisque le séquençage de nouvelle génération rend possible non seulement l'analyse de quelques gènes mais aussi de génomes entiers des organismes du passé augmentant de plusieurs ordres de grandeur la quantité d'informations que l'on peut obtenir. Ceci a permis une révision de notre vision des dernières étapes de l'évolution humaine et du peuplement de l'Eurasie et de l'Amérique, mais aussi des processus de domestication des animaux et de certaines plantes, de l'évolution des populations sauvages animales et végétales en réponse aux changements climatiques, de l'évolution des pathogènes responsables d'épidémies, etc. La paléogénomique a ainsi déjà réussi à révolutionner l'idée que nous nous sommes faite de l'évolution récente de notre espèce, et on peut légitimement attendre d'elle d'autres très belles contributions à l'archéologie.

\begin{abstract}
Paleogenetics, the genetic study of organisms and populations of the past, became possible when the methods of molecular biology allowed the analysis of degraded DNA preserved in trace amounts in certain ancient biological remains, such as bones, teeth, hair or soft tissue of mummies. This has contributed significantly to archeology, paleontology, paleopathology or paleoecology. Recently, the progress of DNA sequencing has brought about a new quantum leap of the field since next-generation sequencing allows the analysis of whole genomes of past organisms. This led to the revision of our view of the last stages of the evolution of humans and of the peopling of Eurasia and the Americas, but also of the domestication of animals and some plants, the evolution of wild populations in response to climate changes, the evolution of pathogens responsible for past epidemics, etc. Paleogenomics revolutionized already the idea we had about the recent evolution of our species, but it is legitimate to expect the field to make further important contributions to archeology.
\end{abstract}

Mots clés : ADN ancien, paléogénétique, paléogénomique, évolution, domestication, paléopathologie.

Keywords: ancient DNA, paleogenetics, paleogenomics, evolution, domestication, paleopathology.

\section{LA GÉNÉTiQUE}

Le généticien étudie et analyse les caractères héréditaires des êtres vivants (micro-organismes, animaux, végétaux, êtres humains) à travers leur génome, l'ensemble du matériel génétique d'un être vivant, dont l'ADN (acide désoxyribonucléique) est le support. Ce matériel génétique est transmis à chaque génération pour assurer la production d'organismes dont l'organisation physique et la physiologie refléteront en grande partie celle des parents. Lorsqu'on analyse la séquence de l'ADN d'un individu, on peut donc ainsi avoir accès à deux grands types d'information : sa généalogie et ses caractères morphologiques et physiologiques. Bien que l'on ait beaucoup d'arguments qui valident le concept que l'ADN code pour les caractères de l'individu, il est encore

a Institut Jacques Monod, Équipe "Epigénome et Paléogénome », UMR 7592, 15, rue Hélène-Brion, 75013 Paris. (eva-maria.geigl@ijm.fr) 
extrêmement complexe d'interpréter correctement comment se fait le passage de la séquence du génome aux propriétés physiques et physiologiques des individus. La compréhension de ce passage est une des grandes questions de la biologie et de la médecine qui mobilisent des centaines des milliers de chercheurs depuis au moins un siècle et pour lequel on ne dispose à l'heure actuelle que de réponses très fragmentaires. Par contre, la généalogie est une question beaucoup plus simple à laquelle on peut commencer à répondre avec très peu d'informations génétiques et pour laquelle on peut avoir une vision fine et détaillée dès que l'on peut accéder à un génome partiel ou complet. Depuis une trentaine d'années, les généticiens ont entrepris d'extraire et d'analyser de l'ADN à partir d'échantillons anciens, et la génétique est devenue une science qui contribue aussi à l'archéologie (figure 1). Je présenterai donc les avancées de la paléogénétique et de la paléogénomique au cours de ces trente dernières années ainsi que l'évolution du métier et des compétences du paléogénéticien. J'aborderai en particulier les questions qui concernent l'archéologie auxquelles ces approches ont pu commencer à apporter un nombre important de réponses.

\section{LA PALÉOGÉNÉTIQUE}

Le domaine s'est développé en 1984 quand un animal taxidermisé vieux d'un siècle a été " génotypé " (Higuchi $e t$ al., 1984). Ce fut le début d'une exploration qui a pris de plus en plus d'ampleur et nous permet, 30 ans après, d'analyser des génomes entiers d'organismes vieux de plusieurs centaines de milliers d'années. Le changement progressif d'échelle et de profondeur temporelle a été le fruit de nombreuses évolutions méthodologiques qui ont résulté d'une part des progrès de la biologie moléculaire et de la génétique et d'autre part de développements méthodologiques spéci-

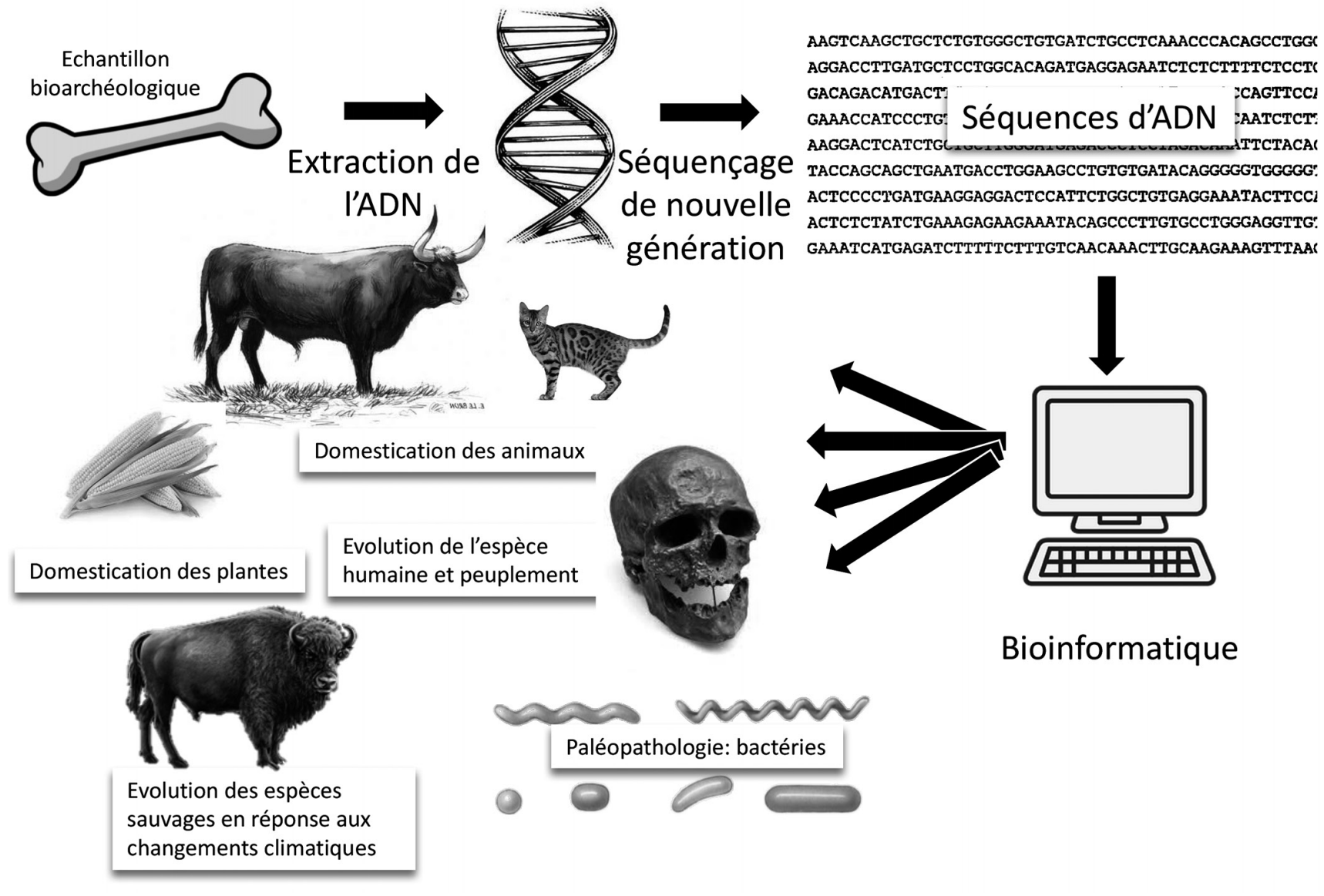

Figure 1 : Représentation schématique du plan de déroulement des analyses paléogénétiques et paléogénomiques montrant des exemples de leur contribution à l'archéologie.

Figure 1: Schematic representation of the workflow of paleogenetic anad paleogenomic analyses showing examples of their contribution to archeo$\log y$. 
fiques au domaine de la paléogénétique. Le premier défi méthodologique qu'il a fallu relever est que l'ADN après la mort est fortement dégradé et qu'il n'en reste donc très vite que des traces sous forme de fragments courts de molécules. La première vague de dégradation est due aux micro- et macroorganismes qui sont responsables de la décomposition des corps et qui éliminent la majeure partie des composants biologiques de l'organisme dont l'ADN. Certaines parties du corps, principalement les parties minéralisées, peuvent persister beaucoup plus longtemps et porter de faibles quantités d'ADN plus ou moins abîmées. Au cours du temps, de multiples attaques chimiques vont affecter cet $\mathrm{ADN}$ résiduel qui ne sera plus réparé après la mort comme il l'était du vivant de l'organisme. Ces attaques vont encore réduire la taille des molécules d'ADN et modifier sa composition d'une façon qui déforme l'information génétique qu'il contient. De plus, cet $\mathrm{ADN}$ est généralement dilué dans une grande quantité d'ADN provenant des microorganismes du sol ayant colonisé le squelette enterré. Il convient aussi de rappeler qu'il faut cribler un nombre considérable d'ossements pour identifier ceux qui contiennent suffisamment d'ADN endogène pour que le génome puisse être déchiffré et qu'une séquence de haute qualité puisse être obtenue.

L'ADN ancien est donc très rare, très abîmé et difficile à analyser. De par sa quantité minime, les molécules d'ADN ancien doivent être multipliées pour être analysées. Ceci s'est fait, entre 1985 et aujourd'hui, avec la technique appelée " $\mathrm{PCR}$ ", une réaction de polymérisation en chaîne enzymatique (Saiki, 1985). C'est une méthode d'une très grande puissance quand elle est optimisée car elle produit des milliards de copies de chaque molécule d'ADN ancienne endogène préservée dans un reste osseux. Par contre, ces copies peuvent facilement contaminer d'autres extraits d'ADN ancien et fausser les résultats des analyses génétiques. C'est pour cela que les premières étapes de l'expérimentation nécessitent un laboratoire de haut-confinement ainsi que le port de combinaisons de protection, de gants et de masques et le respect de procédures de travail très strictes (figure 2). De plus, il faut utiliser des méthodes de décontamination sans lesquelles des résultats erronés peuvent facilement être produits. Ce fléau a été une malédiction du domaine dès sa naissance. Un cas spectaculaire, publié en 1994, qui décrivait l'analyse de l'ADN de dinosaures est l'exemple le plus largement reconnu d'artefact majeur (Woodward et al., 1994). Le problème reste malheureusement suffisamment important pour qu'on puisse dire qu'il existe dans la littérature un certain nombre de résultats partiellement ou complètement erronés et qui sont parfois difficiles à débusquer car toutes les études ne peuvent pas être reproduites.

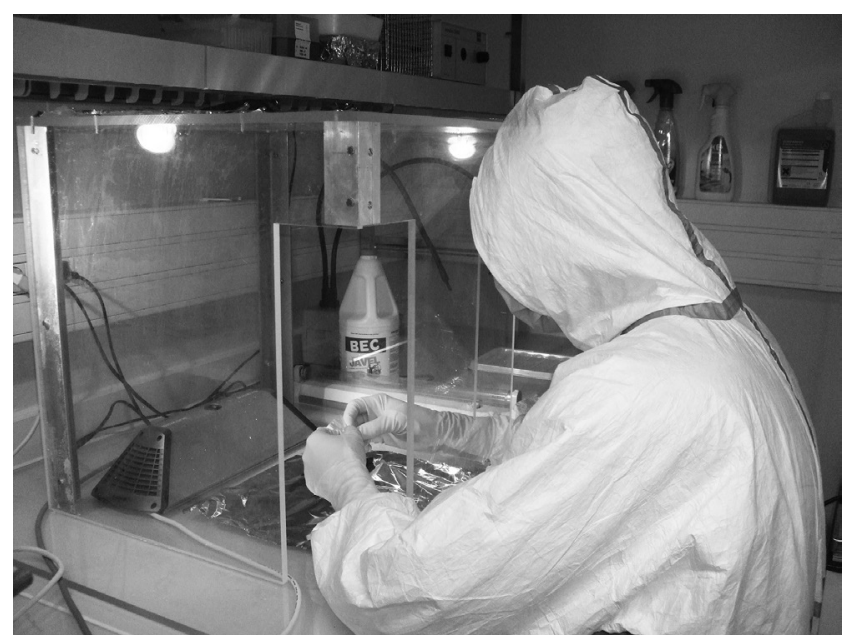

Figure 2 : Analyse de l'ADN préservé dans des ossements archéologiques dans le laboratoire de haut confinement de l'Institut Jacques-Monod (cliché et droit d'auteur : Eva-Maria Geigl). Figure 2: Analysis in the high containment laboratory of the Institut Jacques Monod of DNA preserved in archeological bone.

\section{LES ÉTUdES PALÉOGÉNÉTIQUES}

Malgré ces incertitudes et limites, l'apport de la paléogénétique basé sur les méthodes de PCR à l'archéologie, la paléoanthropologie, l'archéozoologie et l'archéobotanique a été (et est toujours) multiple et riche. Il a permis de déterminer à quelles lignées maternelles ont appartenu des individus mis au jour dans des sites archéologiques et ainsi de reconstruire des migrations et remplacements de populations humaines (par exemple en Europe centrale au cours des dernières 6500 années; Brandt et al., 2013), mais aussi d'animaux domestiqués (par ex : les chats, Ottoni et al., 2017), des relations familiales et sociales au sein des cimetières (par ex : Keyser-Tracqui et al., 2003), identifier des pathogènes et parasites intestinaux (Côté et al., 2016), éclairer les positions phylogénétiques de certains animaux éteints emblématiques (comme le mammouth; Debruyne et al., 2003) ou énigmatiques (comme l'hydrontin; Bennett et al., 2017), déterminer les événements initiaux lors de la domestication des animaux (comme les chevaux; Ludwig et al., 2009), reconstruire le paléoenvironnement grâce à l'analyse de pollens et des sédiments (comme pour le Groenland il y a environ 800 000-450 000 ans; Willerslev et al., 2007) ou encore étudier la réponse de la mégafaune aux changements climatiques et environnementaux et l'action anthropique (Lorenzen et al., 2011). 


\section{La PALÉOgÉnOMIQUe}

Depuis 10 ans environ, les progrès de la biologie moléculaire ont permis de déchiffrer le code génétique de génomes entiers et les améliorations de ces méthodes sont en pleine expansion rendant les études génomiques plus abordables financièrement. Elles produisent des quantités énormes de données qui doivent être traitées avec des méthodes complexes de bioinformatique et de génétique des populations. L'étude de l'analyse de l'ADN ancien a pleinement profité de ces progrès et une nouvelle discipline est née, la paléogénomique qui séquence des génomes entiers, ou des parties de génomes d'organismes du passé. Le métier du paléogénéticien est donc en pleine évolution et demande des compétences non seulement en biologie moléculaire, mais aussi en bioinformatique et en génétique des populations. Du fait que les molécules d'ADN multipliées sont très diverses, contrairement à la PCR ciblée, ces nouvelles méthodes sont moins sensibles à la production de résultats erronés dus à la contamination par l'ADN préalablement multiplié dans le laboratoire. De plus, l'ADN ancien peut être distingué de l'ADN moderne car les patrons de dommages à l'ADN correspondant sont distinguables. Toutefois, ces approches ne sont pas totalement immunes aux contaminations, comme le montre la mise en évidence de contaminations dans la première séquence du génome néanderthalien (Wall et Kim, 2007). Actuellement, ce problème est redevenu une source d'erreurs significatives lorsque la capture de séquences est utilisée à cause de la réduction de la diversité des séquences d'ADN après enrichissement des molécules d'ADN ancien et leur multiplication par PCR. Il est donc très important de garder un œil critique quand on analyse les données publiées pour savoir si les conclusions tirées et les interprétations qui en découlent sont justifiées.

Cette révolution paléogénomique a radicalement augmenté l'importance de la contribution de la génétique à l'archéologie et aux archéosciences biologiques. Elle a notamment permis de modifier notre vision de l'évolution humaine depuis 1 million d'années et de réécrire certaines de ses étapes. Le séquençage des génomes des Néanderthaliennes de l'Altaï (Sibérie) et de Vindija (Croatie) a permis de démontrer l'existence de flux génique entre des Néanderthaliens et humains anatomiquement modernes venant de quitter l'Afrique en plusieurs vagues. Un premier événement de métissage, sans descendance moderne, avait lieu il y a environ 100000 ans et au moins un deuxième il y a $47000-65000$ ans (Kuhlwilm et al., 2016; Prüfer et al., 2017; Prüfer et al., 2014). Suite à ces métissages, certaines régions génomiques d'origine néanderthalienne ont été éli- minées très tôt, probablement parce qu'elles réduisaient la fécondité de la descendance, tandis que d'autres régions ont été conservées, probablement parce qu'elles donnaient un avantage sélectif, au moins dans le passé (Fu et al., 2015). Ainsi tous les Eurasiatiques actuels portent toujours environ $2 \%$ d'ADN néanderthalien dans leurs génomes, témoin de ce métissage (Sankararaman et al., 2014). Certains des variants géniques d'origine néanderthalienne ont dû conférer un avantage sélectif aux métis lors de la colonisation de nouveaux territoires où ils ont rencontré de nouveaux pathogènes et où ils ont dû s'adapter à un autre environnement (Fumagalli et Sironi, 2014). En effet, l'incorporation des variants géniques de populations archaïques autochtones qui se sont adaptées pendant des dizaines de milliers d'années aux conditions de leur habitat a été certainement avantageux pour les nouveaux-arrivants (Dannemann et Kelso, 2017; Simonti et al., 2016).

\section{LA PALÉOgÉNOMIQUE POUR ÉTUdIER L'ÉVOLUTION HUMAINE}

La lecture des génomes anciens nous permet ainsi de reconstruire le chemin de notre évolution, d'identifier des événements de métissage et même de lignées humaines inconnues aux paléoanthropologues, comme c'est le cas des Dénisoviens, cette population asiatique à laquelle on ne peut (pas encore) associer un squelette, à part des molaires, mais dont on dispose d'un génome d'une très grande qualité obtenu à partir d'un minuscule fragment de la base de la cinquième phalange de la main (Meyer et al., 2012). Ce génome dont l'analyse s'étalera encore sur plusieurs années, nous a déjà fourni et fournira encore énormément d'informations non seulement sur cet individu mais aussi sur la population à laquelle elle appartenait ainsi qu'à son histoire évolutive et celle des êtres humains modernes. La comparaison de ces génomes archaïques avec les génomes des populations modernes a permis de déceler et dater les événements de métissage et d'en déduire des migrations de différentes populations humaines. Là aussi, le métissage a permis aux populations d'êtres humains migrants de s'adapter à des nouveaux environnements, comme par exemple cela a été montré pour l'adaptation à l'altitude des Tibétains qui proviendrait en partie de variants génétiques d'origine dénisovienne (Huerta-Sanchez et al., 2014).

L'analyse des génomes des populations anciennes ne s'est pas arrêtée au Paléolithique. Il a été possible de séquencer des génomes d'individus de différentes périodes et cultures et de les caractériser génétiquement. Ceci a permis d'identifier de nombreuses vagues de migrations depuis le dernier 
maximum glaciaire en comparant les génomes d'individus associés au Paléolithique supérieur, puis au Mésolithique, au Néolithique et à l'Âge du Bronze. Ainsi il a été possible d'identifier les composants principaux du génome des Européens actuels (Fu et al., 2016; Lazaridis et al., 2014). La première composante est africaine et provient des humains anatomiquement modernes de l'Afrique ayant colonisé l'Asie, il y a -65000 ans, et l'Europe, il y a -43000 ans. Par contre, la première branche de ces Proto-Européens représente un cul-de-sac car elle n'a pas laissé beaucoup de matériel génétique dans les Européens actuels (Fu et al., 2014; Fu et al., 2016). Une autre branche a évolué en Europe, alors qu'une troisième branche a évolué en Europe de l'Est. Tandis que la première population, plus ancienne, pourrait être celle qui a développé la culture aurignacienne, la branche de l'Europe de l'Est pourrait être associée à la culture gravettienne qui s'est répandue en Europe sans que la population associée à la culture aurignacienne n'ait été remplacée (Fu et al., 2016). Cette dernière a continué à évoluer et aurait finalement produit la culture magdalénienne. C'est seulement à la fin de la glaciation, avec le réchauffement climatique, qu'une population du Proche-Orient est venue par l'Europe du Sud-Est, a remplacé les populations autochtones de la culture magdalénienne et épigravettienne et aurait développé la culture mésolithique (Fu et al., 2016). Les premiers agriculteurs de l'Anatolie, descendants d'un métissage entre deux populations distinctes originaires du Levant et de l'Iran (Lazaridis et al., 2016) ont diffusé le Néolithique en colonisant l'Europe il y a -7000 ans par deux vagues de migrations, le courant continental et le courant méditerranéen, et qui ont emmené les premiers animaux et plantes qu'ils avaient domestiqués, comme l'attestent les données archéologiques mais aussi paléogénétiques et paléogénomiques (voir plus loin). À la fin du Néolithique, début de l'Âge du Bronze, les populations des nomades des steppes pontiques de la culture des Kourganes ("Yamna " en russe), descendants de métis entre une population ayant survécu à la dernière glaciation dans le Caucase de chasseurs-cueilleurs du territoire russe et d'agriculteurs néolithiques d'Iran se sont rapidement disséminés en Europe centrale sur le dos des chevaux qu'ils avaient domestiqués (Allentoft et al., 2015; Haak et al., 2015; Lazaridis et al., 2016). Leur succès reproductif sur le territoire de l'Allemagne du Nord a été très supérieur à celui des populations autochtones car les générations suivantes ont hérité jusqu'à $80 \%$ de leur génome de la population migrante (Haak et al., 2015), voir aussi figure 3. Les données génomiques livrent donc une trame dans laquelle il est possible de placer les données archéologiques et linguistiques. En revanche, pour pouvoir bien interpréter les données génomiques, il faut tenir compte des données archéologiques et une collaboration étroite entre paléogénéticiens et archéologues s'impose. Ceci est vrai pour tous les projets en paléogénétique basés sur du matériel issu de fouilles archéologiques.

\section{LA PALÉOgÉNOMIQUE DES ANIMAUX ET PLANTES}

Les progrès du séquençage et de l'analyse des génomes entiers ne se restreignent pas à l'analyse des génomes humains, mais concernent aussi les génomes des animaux et plantes. Ainsi il a été possible d'étudier la dynamique des populations d'animaux liée aux changements climatiques et environnementaux, comme par exemple des bisons en Europe (Massilani et al., 2016), ce qui a aussi permis d'identifier une espèce qui n'était pas connue à cette époque en France, un bison européen ancien. La paléogénomique a, de manière générale, le potentiel d'aider les paléontologues pour l'identification des espèces. Un travail important a été fait sur le genre Equus ce qui a eu comme conséquence de simplifier la taxonomie (Orlando et al., 2013).

L'analyse des génomes des animaux au cours de leur domestication est aussi un sujet de prédilection de la paléogénomique et a déjà porté de nombreux fruits, comme par exemple la démonstration de l'existence de deux foyers de domestication du chien (Frantz et al., 2016).

La paléogénomique ne s'arrête pas seulement aux ossements mais peut aussi analyser les génomes des bactéries impliquées dans des pathologies, comme la peste (Bos et al., 2011) ou la tuberculose (Bos et al., 2014), ou bien des bactéries, plantes et animaux préservés dans les sédiments (Pedersen et al., 2015). Des séquences d'ADN bactériennes, animales et végétales sont aussi très bien préservées dans le tartre dentaire et peuvent témoigner de maladies buccales et systémiques et de l'alimentation des individus (Warinner et al., 2014).

\section{Conclusion et Perspectives}

La quantité d'information que pourra fournir la séquence d'un génome de haute qualité, comme ceux des Néandertaliennes d'Altaï et de Vindija ainsi que de la Dénisovienne, est immense et souvent encore mal appréciée par les anthropologues, paléontologues, archéologues et conservateurs. En effet, l'information que la communauté des généticiens est capable de récupérer à partir de la séquence d'un génome ne représente encore qu'une partie de ce qui est codé dans ce génome. Comme expliqué auparavant, on peut déjà très bien caractériser les liens de parenté, 


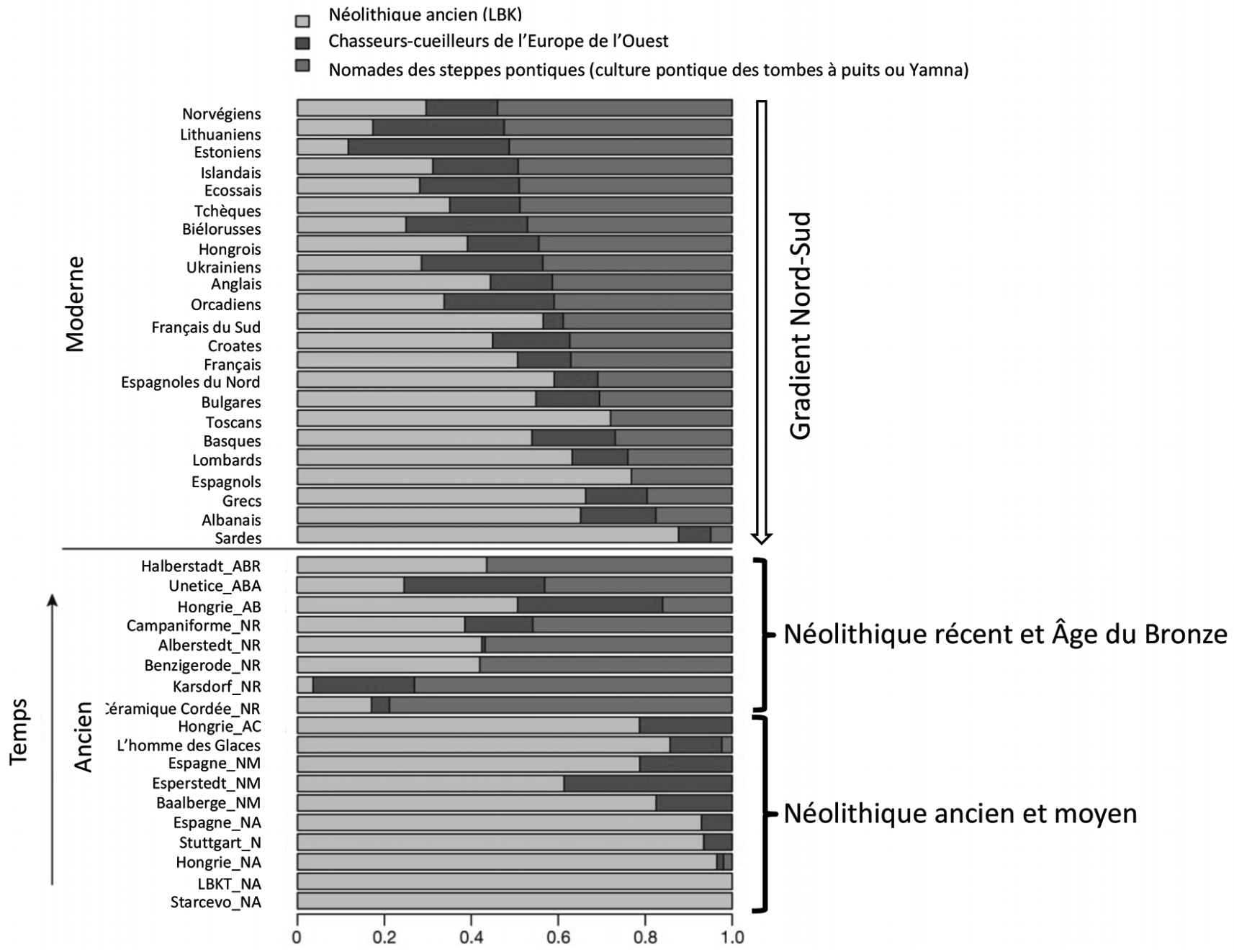

Figure 3 : (Voir planche couleur XVI) L'analyse de presque 400000 mutations dispersées sur les génomes d'une centaine d'Européens anciens préservés dans des contextes archéologiques néolithiques, chalcolithiques, et de l'âge du Bronze ainsi que dans les populations européennes actuelles (Haak et al., 2015). L'analyse STRUCTURE révèle les différentes parties du génome originaires des chasseurs-cueilleurs mésolithiques d'Europe de l'Ouest, des premiers agriculteurs néolithiques ainsi que des nomades des steppes pontiques de la culture Yamna présentes dans chaque génome (Haak et al., 2015). Il ressort de cette analyse que l'ascendance des premiers populations néolithiques originaires du Proche-Orient est plus forte dans les populations actuelles de l'Europe du Sud que dans le Nord alors que, inversement, l'ascendance des peuples des steppes pontiques est plus forte dans les populations actuelles de l'Europe du Nord. Cela nous renseigne sur les endroits et directions des métissages entre populations autochtones et populations migrantes. ( $\mathrm{N}=$ Néolithique; NA = Néolithique ancien; $\mathrm{NM}=$ Néolithique moyen; $\mathrm{NR}=$ Néolithique récent; $\mathrm{AC}=$ Âge du Cuivre; $\mathrm{ABA}=$ Âge du Bronze ancien; $\mathrm{ABR}=\hat{\mathrm{A} g e} \mathrm{du} \mathrm{Bronze}$ récent). Figure 3: (See colour plate XVI) The genome-wide analysis of almost 400,000 mutations in about hundred ancient European genomes preserved in Neolithic, chalcolithic and Bronze Age archeological contexts and in modern European populations (Haak et al., 2015). STRUCTURE analysis shows the different components of each genome presumably stemming from Mesolithic hunter-gatherers in West Europe, the first Neolithic farmers and the nomades from the Pontic Steppes (Yamnaya) (Haak et al., 2015). This analysis demonstrates that the ancestry of the first Neolithic populations coming from the Near East is stronger in the present-day populations of South Europe than in the North. Moreover, it shows that the ancestry from the nomades of the Pontic Steppes is stronger in the present-day populations from North Europe. This informs about the locations and directions of the admixture between authochtonous and migrating populations. ( $N=$ Neolithic; $N A=$ Early Neolithic; NM = Middle Neolithic; $N R=$ Late Neolithic; $A C=$ Copper Age; $A B A=$ Early Bronze Age; ABR = Late Bronze Age).

les effets démographiques, certains événements de métissage, ainsi que quelques gènes dont les voies de synthèse biochimique et l'action des protéines produites sont connues. Par contre, jusqu'à aujourd'hui, il reste encore beaucoup de gènes qui ne sont pas entièrement analysés chez les êtres humains actuels, et encore moins chez les animaux et plantes, bien 
que les dizaines de milliers de chercheurs en génétique qui s'attellent à ce déchiffrement des liens entre génotype et phénotype font progresser régulièrement la connaissance. Or, les paléogénomiciens ne peuvent analyser dans les génomes anciens que les mutations dont on connaît l'action. C'est pour cela que dans les années à venir, les généticiens seront à même de réanalyser les génomes anciens dont la séquence est connue, accessible pour tout chercheur, c'est-à-dire immortalisée, pour extraire de plus en plus d'informations sur la biologie des populations anciennes suite aux progrès que feront la biologie et génétique fondamentale, médicale, animale et végétale. Ainsi, le patrimoine génétique des restes biologiques anciens qui constitue un patrimoine au moins aussi important que leur patrimoine morphologique grâce à la quantité immense d'information qu'il contient, n'est qu'au début de son exploration et progresse avec des nouvelles découvertes qui se font quotidiennement, livrant petit à petit ses secrets.

Grâce au développement rapide des techniques de séquençage permettant une augmentation importante de la quantité de séquences produites, le domaine de la paléogénomique a donc encore de belles années devant elle. C'est un domaine attractif pour les étudiants et le nombre de docteurs en paléogénétique et paléogénomique est en pleine croissance. Les étudiants doivent acquérir des compétences pointues en biologie moléculaire et en bioinformatique ce qui place le vivier de recrutement plutôt du côté des biologistes que des archéologues. Toutefois une formation complémentaire en archéologie s'adressant à ce corps de métier serait bénéfique car elle faciliterait la discussion avec les archéologues, la mise en place de protocoles de prélèvement et la collaboration avec des collègues des pays où l'exportation d'échantillons est interdite, contribuant ainsi à une meilleure interprétation des résultats. Cette évolution permettra d'augmenter encore la contribution de ce domaine à l'archéologie et à notre connaissance de l'évolution des humains et des sociétés pré-, proto- et historiques, de la biodiversité du passé, de la paléoécologie et de la domestication des animaux et plantes.

\section{Bibliographie}

Allentoft M.E., Sikora M., Sjogren K.G., Rasmussen S., Rasmussen M., Stenderup J., Damgaard P.B., Schroeder H., Аhlstrom T., Vinner L., Malaspinas A.S., Margaryan A., Higham T., Chivall D., Lynnerup N., Harvig L., Baron J., Della Casa P., Dabrowski P., Duffy P.R., Ebel A.V., Epimakhov A., Frei K., Furmanek M., Gralak T., Gromov A., Gronkiewicz S., Grupe G., Hajdu T., Jarysz
R., Khartanovich V., Khokhlov A., Kiss V., Kolar J., Krisska A., Lasak I., Longhi C., McGlynn G., Merkevicius A., Merkyte I., Metspalu M., Mkrtchyan R., Moiseyev V., Paja L., Palfi G., Pokutta D., Pospieszny L., Price T.D., SaAg L., Sablin M., Shishlina N., Smrcka V., Soenov V.I., Szeverenyi V., Toth G., Trifanova S.V., Varul L., Vicze M., Yepiskoposyan L., Zhitenev V., Orlando L., Sicheritz-Ponten T., Brunak S., Nielsen R., Kristiansen K., Willerslev E., 2015. Population genomics of Bronze Age Eurasia. Nature, 522, p. 167-172.

Bennett E.A., Champlot S., Peters J., Arbuckle B.S., Guimaraes S., Pruvost M., Bar-David S., Davis S. J.M., Gautier M., Kaczensky P., Kuehn R., Mashkour M., Morales-Muniz A., Pucher E., Tournepiche J.F., Uerpmann H.P., Balasescu A., Germonpre M., Gundem C.Y., Hemami M.R., Moulle P.E., Otzan A., Uerpmann M., Walzer C., Grange T., Geigl E.M., 2017. Taming the late Quaternary phylogeography of the Eurasiatic wild ass through ancient and modern DNA. PLoS one, 12: e0174216.

Bos K. I., Harkins K.M., Herbig A., Coscolla M., Weber N., Comas I., Forrest S.A., Bryant J. M., Harris S. R., Schuenemann V.J., Campbell T.J., Majander K., Wilbur A.K., Guichon R. A., Wolfe Steadman D.L., Cook D.C., Niemann S., Behr M.A., Zumarraga M., Bastida R., Huson D., Nieselt K., Young D., Parkhill J., Buikstra J.E., Gagneux S., Stone A.C., Krause J., 2014. Pre-Columbian mycobacterial genomes reveal seals as a source of New World human tuberculosis. Nature, 514(7523), p. 494-497.

Bos K. I., Schuenemann V.J., Golding G.B., Burbano H.A., Waglechner N., Coombes B.K., McPhee J.B., DeWitte S.N., Meyer M., Schmedes S., Wood J., Earn D.J., Herring D.A., Bauer P., Poinar H.N., Krause J., 2011. A draft genome of Yersinia pestis from victims of the Black Death. Nature, 478, p. 506-510.

Brandt G., Haak W., Adler C.J., Roth C., Szecsenyi-Nagy A., Karimnia S., Moller-Rieker S., Meller H., Ganslmeier R., Friederich S., Dresely V., Nicklisch N., Pickrell J.K., Sirocko F., Reich D., Cooper A., Alt K.W., 2013. Ancient DNA reveals key stages in the formation of central European mitochondrial genetic diversity. Science, 342, p. 57-261.

Côté N. M., Daligault J., Pruvost M., Bennett E.A., Gorgé O., Guimaraes S., Capelli N., Le Bailly M., Geigl E.M., Grange T., 2016. A New High-Throughput Approach to Genotype Ancient Human Gastrointestinal Parasites. PLoS one, 11: e0146230.

Dannemann M., Kelso J., 2017. The Contribution of Neanderthals to Phenotypic Variation in Modern Humans. Am J Hum Genet 101, p. 578-589.

Debruyne R., Barriel V., Tassy P., 2003. Mitochondrial cytochrome b of the Lyakhov mammoth (Proboscidea, Mammalia): 
new data and phylogenetic analyses of Elephantidae. Molecular Phylogenetics and Evolution, 26, p. 421-434.

Frantz L.A., Mullin V.E., Pionnier-Capitan M., Lebrasseur O., Ollivier M., Perri A., Linderholm A., Mattiangeli V., Teasdale M.D., Dimopoulos E.A., Tresset A., Duffraisse M., McCormick F., Bartosiewicz L., Gal E., Nyerges E.A., Sablin M.V., Brehard S., Mashkour M., Balasescu A., Gillet B., Hughes S., Chassaing O., Hitte C., Vigne J.D., Dobney K., Hanni C., Bradley D.G., Larson G., 2016. Genomic and archaeological evidence suggest a dual origin of domestic dogs. Science, 352, p. 1228-1231.

Fu Q., Hajdinjak M., Moldovan O.T., Constantin S., Mallick S., Skoglund P., Patterson N., Rohland N., Lazaridis I., Nickel B., Viola B., Prufer K., Meyer M., Kelso J., Reich D., Paabo S., 2015. An early modern human from Romania with a recent Neanderthal ancestor. Nature, 524, p. 216-219.

Fu Q., Li H., Moorjani P., Jay F., Slepchenko S.M., Bondarev A.A., Johnson P.L., Aximu-Petri A., Prufer K., de Filippo C., Meyer M., Zwyns N., Salazar-Garcia D.C., Kuzmin Y.V., Keates S.G., Kosintsev P.A., Razhev D.I., Richards M.P., Peristov N.V., Lachmann M., Douka K., Higham T.F., Slatkin M., Hublin J.J., Reich D., Kelso J., Viola T.B., PAABo S., 2014. Genome sequence of a 45,000-year-old modern human from western Siberia. Nature, 514, p. 445-449.

Fu Q., Posth C., Hajdinjak M., Petr M., Mallick S., Fernandes D., Furtwangler A., HaAk W., Meyer M., Mittnik A., Nickel B., Peltzer A., Rohland N., Slon V., Talamo S., Lazaridis I., Lipson M., Mathieson I., Schiffels S., Skoglund P., Derevianko A.P., Drozdov N., Slavinsky V., Tsybankov A., Cremonesi R.G., Mallegni F., Gely B., Vacca E., Morales M.R., Straus L. G., Neugebauer-Maresch C., Teschler-Nicola M., Constantin S., Moldovan O.T., Benazzi S., Peresani M., Coppola D., Lari M., Ricci S., Ronchitelli A., Valentin F., Thevenet C., Wehrberger K., Grigorescu D., Rougier H., Creveceeur I., Flas D., Semal P., Mannino M.A., Cupillard C., Bocherens H., Conard N.J., Harvati K., Moiseyev V., Drucker D.G., Svoboda J., Richards M.P., Caramelli D., Pinhasi R., Kelso J., Patterson N., Krause J., Paabo S., Reich D., 2016. The genetic history of Ice Age Europe. Nature, 534, p. 200-205.

Fumagalli M., Sironi M., 2014. Human genome variability, natural selection and infectious diseases. Current Opinion in Immunology, 30, p. 9-16.

Haak W., Lazaridis I., Patterson N., Rohland N., Mallick S., Llamas B., Brandt G., Nordenfelt S., Harney E., Stewardson K., Fu Q., Mittnik A., Banffy E., Economou C., Francken M., Friederich S., Pena R.G., Hallgren F., Khartanovich V., Khokhlov A., Kunst M., Kuznetsov
P., Meller H., Mochalov O., Moiseyev V., Nicklisch N., Pichler S.L., Risch R., Rojo Guerra M.A., Roth C., Szecsenyi-Nagy A., Wahl J., Meyer M., Krause J., Brown D., Anthony D., Cooper A., Alt K.W., Reich D., 2015. Massive migration from the steppe was a source for IndoEuropean languages in Europe. Nature, 522, p. 207-211.

Higuchi R., Bowman B., Freiberger M., Ryder O.A., Wilson A.C., 1984. DNA sequences from the quagga, an extinct member of the horse family. Nature, 312, p. 282-284.

Huerta-Sanchez E., Jin X., Asan, Bianba Z., Peter B.M., Vinckenbosch N., Liang Y., Yi X., He M., Somel M., Ni P., Wang B., Ou X., Huasang, Luosang J., Cuo Z.X., Li K., GaO G., Yin Y., Wang W., Zhang X., Xu X., Yang H., Li Y., Wang J., Nielsen R., 2014. Altitude adaptation in Tibetans caused by introgression of Denisovan-like DNA. Nature, 512, p. 194-197.

Keyser-Tracqui C., Crubézy E., Ludes B., 2003. Nuclear and mitochondrial DNA analysis of a 2,000-year-old necropolis in the Egyin Gol Valley of Mongolia. American Journal of Human Genetics, 73, p. 247-260.

Kuhlwilm M., Gronau I., Hubisz M.J., de Filippo C., PradoMartinez J., Kircher M., Fu Q., Burbano H.A., LaluezaFox C., de la Rasilla M., Rosas A., Rudan P., Brajkovic D., Kucan Z., Gusic I., Marques-Bonet T., Andres A.M., Viola B., Paabo S., Meyer M., Siepel A., Castellano S., 2016. Ancient gene flow from early modern humans into Eastern Neanderthals. Nature, 530(7591), p. 429-433.

Lazaridis I., Nadel D., Rollefson G., Merrett D.C., Rohland N., Mallick S., Fernandes D., Novak M., Gamarra B., Sirak K., Connell S., Stewardson K., Harney E., Fu Q., Gonzalez-Fortes G., Jones E.R., Roodenberg S.A., Lengyel G., Bocquentin F., Gasparian B., Monge J. M., Gregg M., Eshed V., Mizrahi A.S., Meiklejohn C., Gerritsen F., Bejenaru L., Bluher M., Campbell A., Cavalleri G., Comas D., Froguel P., Gilbert E., Kerr S.M., Kovacs P., Krause J., McGettigan D., Merrigan M., Merriwether D.A., O'Reilly S., Richards M.B., Semino O., Shamoon-Pour M., Stefanescu G., Stumvoll M., Tonjes A., Torroni A., Wilson J.F., Yengo L., Hovhannisyan N.A., Patterson N., Pinhasi R., Reich D., 2016. Genomic insights into the origin of farming in the ancient Near East. Nature, 536, p. 419-424.

Lazaridis i., Patterson N., Mittnik A., Renaud G., Mallick S., Kirsanow K., Sudmant P.H., Schraiber J. G., Castellano S., Lipson M., Berger B., Economou C., Bollongino R., Fu Q., Bos K. I., Nordenfelt S., Li H., de Filippo C., Prufer K., Sawyer S., Posth C., Haak W., Hallgren F., Fornander E., Rohland N., Delsate D., Francken M., Guinet J. M., Wahl J., Ayodo G., Babiker H.A., Bailliet G., Balanovska E., Balanovsky 
O., Barrantes R., Bedoya G., Ben-Ami H., Bene J., Berrada F., Bravi C.M., Brisighelli F., Busby G.B., Cali F., Churnosov M., Cole D.E., Corach D., Damba L., van Driem G., Dryomov S., Dugoujon J.M., Fedorova S.A., Gallego Romero I., Gubina M., Hammer M., Henn B.M., Hervig T., Hodoglugil U., Jha A.R., KarachanaKYankova S., Khusainova R., Khusnutdinova E., Kittles R., Kivisild T., Klitz W., Kucinskas V., Kushniarevich A., Laredj L., Litvinov S., Loukidis T., Mahley R.W., Melegh B., Metspalu E., Molina J., Mountain J., Nakkalajarvi K., Nesheva D., Nyambo T., Osipova L., Parik J., Platonov F., Posukh O., Romano V., Rothhammer F., Rudan I., Ruizbakiev R., Sahakyan H., Sajantila A., Salas A., Starikovskaya E.B., Tarekegn A., Toncheva D., Turdikulova S., Uktveryte I., Utevska O., Vasquez R., Villena M., Voevoda M., Winkler C.A., Yepiskoposyan L., Zalloua P., Zemunik T., Cooper A., Capelli C., Thomas M.G., Ruiz-Linares A., Tishkoff S.A., Singh L., Thangaraj K., Villems R., Comas D., Sukernik R., Metspalu M., Meyer M., Eichler E.E., Burger J., Slatkin M., Paabo S., Kelso J., Reich D., Krause J., 2014. Ancient human genomes suggest three ancestral populations for present-day Europeans. Nature 513, p. 409-413.

Lorenzen E.D., Nogues-Bravo D., Orlando L., Weinstock J., Binladen J., Marske K.A., Ugan A., Borregatrd M.K., Gilbert M.T., Nielsen R., Ho S.Y., Goebel T., Graf K.E., Byers D., Stenderup J.T., Rasmussen M., Campos P.F., Leonard J.A., Koepfli K.P., Froese D., Zazula G., Stafford T.W., Jr., Aaris-Sorensen K., Batra P., Haywood A.M., Singarayer J.S., Valdes P. J., Boeskorov G., Burns J.A., Davydov S.P., Haile J., Jenkins D.L., Kosintsev P., Kuznetsova T., Lai X., Martin L.D., McDonald H.G., Mol D., Meldgaard M., Munch K., Stephan E., Sablin M., Sommer R.S., Sipko T., Scott E., Suchard M.A., Tikhonov A., Willerslev R., Wayne R.K., Cooper A., Hofreiter M., Sher A., Shapiro B., Rahbek C., Willerslev E., 2011. Species-specific responses of Late Quaternary megafauna to climate and humans. Nature 479, p. 359-364.

Ludwig A., Pruvost M., Reissmann M., Benecke N., Brockmann G.A., Castanos P., Cieslak M., Lippold S., Llorente L., Malaspinas A.S., Slatkin M., Hofreiter M., 2009. Coat color variation at the beginning of horse domestication. Science 324, p. 485.

Massilani D., Guimaraes S., Brugal J.P., Bennett E. A., Tokarska M., Arbogast R.M., Baryshnikov G., Boeskorov G., Castel J.C., Davydov S., Madelaine S., Putelat O., Spasskaya N.N., Uerpmann H.P., Grange T., Geigl E.M., 2016. Past climate changes, population dynamics and the origin of Bison in Europe. BMC Biology, 14, p. 93-110.
Meyer M., Kircher M., Gansauge M.T., Li H., Racimo F., Mallick S., Schraiber J.G., Jay F., Prufer K., de Filippo C., Sudmant P.H., Alkan C., Fu Q., Do R., Rohland N., Tandon A., Siebauer M., Green R.E., Bryc K., Briggs A.W., Stenzel U., Dabney J., Shendure J., Kitzman J., Hammer M.F., Shunkov M.V., Derevianko A.P., Patterson N., Andres A.M., Eichler E.E., Slatkin M., Reich D., Kelso J., Раabo S., 2012. A high-coverage genome sequence from an archaic Denisovan individual. Science, 338, p. 222226.

Orlando L., Ginolhac A., Zhang G., Froese D., Albrechtsen A., Stiller M., Schubert M., Cappellini E., Petersen B., Moltke I., Johnson P.L., Fumagalli M., Vilstrup J.T., Raghavan M., Korneliussen T., Malaspinas A.S., Vogt J., Szklarczyk D., Kelstrup C.D., Vinther J., Dolocan A., Stenderup J., Velazquez A.M., Cahill J., Rasmussen M., Wang X., Min J., Zazula G.D., Seguin-Orlando A., Mortensen C., Magnussen K., Thompson J. F., Weinstock J., Gregersen K., Roed K.H., Eisenmann V., Rubin C.J., Miller D.C., Antczak D.F., Bertelsen M. F., Brunak S., Al-Rasheid K.A., Ryder O., Andersson L., Mundy J., Krogh A., Gilbert M.T., Kjaer K., Sicheritz-Ponten T., Jensen L.J., Olsen J.V., Hofreiter M., Nielsen R., Shapiro B., Wang J., Willerslev E., 2013. Recalibrating Equus evolution using the genome sequence of an early Middle Pleistocene horse. Nature, 499, p. 74-78.

Ottoni C., Van Neer W., De Cupere B., Daligault J., Guimaraes S., Peters J., Spassov N., Prendergast M.E., Boivin N.L., Morales-Muñiz A., Balasescu A., Benecke N., Boroneanț A., Buitenhuis H., Chahoud J., Crowther A., Llorente L., Manaseryan N., Monchot H., Onar V., Osypińska M., Putelat O., Quintana Morales E.M., Studer J., Wierer U., Decorte R., Grange T., Geigl E.M., 2017. The palaeogenetics of cat dispersal in the ancient world. Nature Ecology and Evolution, 1, 0139.

Pedersen A.G., Overballe-Petersen S., Ermini L., Der Sarkissian C., Haile J., Hellstrom M., Spens J., Thomsen P.F., Bohmann K., Cappellini E., Baerholm Schnell I., Wales N.A., Caroe C., Campos P.F., Schmidt A.M.Z., Gilbert M.T.P., Hansen A.J., Orlando L., Willerslev E., 2015. Ancient and modern environmental DNA. Philosophical Transactions of the Royal Society B: Biological Sciences, 370: 20130383

Prüfer K., de Filippo C., Grote S., Mafessoni F., Korlevic P., Hajdinjak M., Vernot B., Skov L., Hsieh P., Peyregne S., Reher D., Hopfe C., Nagel S., Maricic T., Fu Q., Theunert C., Rogers R., Skoglund P., Chintalapati M., Dannemann M., Nelson B.J., Key F.M., Rudan P., Kucan Z., Gusic I., Golovanova L.V., Doronichev V.B., Patterson N., Reich D., Eichler E. E., Slatkin M., 
Schierup M.H., Andres A.M., Kelso J., Meyer M., PäÄвo S., 2017. A high-coverage Neandertal genome from Vindija Cave in Croatia. Science, 358, p. 655-658.

Prüfer K., Racimo F., Patterson N., Jay F., Sankararaman S., Sawyer S., Heinze A., Renaud G., Sudmant P.H., de Filippo C., Li H., Mallick S., Dannemann M., Fu Q., Kircher M., Kuhlwilm M., Lachmann M., Meyer M., Ongyerth M., Siebauer M., Theunert C., Tandon A., Moorjani P., Pickrell J., Mullikin J.C., Vohr S.H., Green R.E., Hellmann I., Johnson P.L., Blanche H., Cann H., Kitzman J.O., Shendure J., Eichler E.E., Lein E.S., Bakken T.E., Golovanova L. V., Doronichev V. B., Shunkov M. V., Derevianko A.P., Viola B., Slatkin M., Reich D., Kelso J., РӓÄво S., 2014. The complete genome sequence of a Neanderthal from the Altai Mountains. Nature, 505, p. 43-49.

Saiki R. K., 1985. Enzymatic amplification of b-globin genomic sequences and restriction site analysis for diagnosis of sickle cell anemia. Science, 230, p. 1350-1354.

Sankararaman S., Mallick S., Dannemann M., Prufer K., Kelso J., Paabo S., Patterson N., Reich D., 2014. The genomic landscape of Neanderthal ancestry in present-day humans. Nature, 507, p. 354-357.

Simonti C. N., Vernot B., Bastarache L., Bottinger E., Carrell D.S., Chisholm R.L., Crosslin D.R., Hebbring S. J., Jarvik G.P., Kullo I. J., Li R., Pathak J., Ritchie M.D., Roden D.M., Verma S.S., Tromp G., Prato J.D.,
Bush W.S., Akey J.M., Denny J. C., Capra J.A., 2016. The phenotypic legacy of admixture between modern humans and Neandertals. Science, 351, p. 737-741.

Wall J.D., Kim S.K., 2007. Inconsistencies in Neanderthal genomic DNA sequences. PLoS Genet, 3, p. 1862-1866.

Warinner C., Rodrigues J. F., Vyas R., Trachsel C., Shved N., Grossmann J., Radini A., Hancock Y., Tito R.Y., Fiddyment S., Speller C., Hendy J., Charlton S., Luder H.U., Salazar-Garcia D. C., Eppler E., Seiler R., Hansen L.H., Castruita J. A., Barkow-Oesterreicher S., Teoh K.Y., Kelstrup C.D., Olsen J. V., Nanni P., Kawai T., Willerslev E., von Mering C., Lewis C.M., Jr., Collins M.J., Gilbert M.T., Ruhli F., Cappellini E., 2014. Pathogens and host immunity in the ancient human oral cavity. Nature Genetics, 46, p. 336-344.

Willerslev E., Cappellini E., Boomsma W., Nielsen R., Hebsgaard M. B., Brand T.B., Hofreiter M., Bunce M., Poinar H.N., Dahl-Jensen D., Johnsen S., Steffensen J.P., Bennike O., Schwenninger J.L., Nathan R., Armitage S., de Hoog C.J., Alfimov V., Christl M., Beer J., Muscheler R., Barker J., Sharp M., Penkman K.E., Haile J., Taberlet P., Gilbert M.T., Casoli A., Campani E., Collins M.J., 2007. Ancient biomolecules from deep ice cores reveal a forested southern Greenland. Science 317, p. 111-114.

Woodward S.R., Weyand N.J., Bunnell M., 1994. DNA sequence from Cretaceous period bone fragments. Science, 266, p. $1229-1232$. 